\title{
ESTUDO DE FALHAS DE PROCESSOS DE GERENCIAMENTO DE ALARMES BASEADO ÍNDICES DE DESEMPENHO E ANÁLISE DE CAUSA-RAIZ*
}

Eduardo Montenegro ${ }^{1}$

\section{Resumo}

Apesar da relevância e interesse do tema de gerenciamento de alarmes na comunidade técnica industrial, observa-se que poucas empresas têm, de fato, implantado e conseguido extrair melhores resultados na eficiência e segurança operacional de suas plantas. O presente trabalho visa, a partir das experiências dos autores em implantação e acompanhamento de vários sistemas de gerenciamento de alarmes na indústria, discutir os principais motivos pelos quais estes sistemas falham em conseguir índices melhores em operação, monitoramento e gestão de alarmes. Além disso, propõe-se neste trabalho que estratégias, práticas e ferramentas sejam adotadas para que se consiga o máximo de benefício operacional ao se implantar e manter tais sistemas.

Palavras-chave: Gerenciamento de alarmes; Análise de causa-raiz; Sistemas SCADA.

\section{STUDY OF PROCESS FAULTS IN ALARMS MANAGEMENT BASED PERFORMANCE INDICATORS AND ANALYSIS OF ROOT CAUSE}

\section{Abstract}

Despite of the relevance and interest of the theme alarm management in technical industrial community, a few industries have, in fact, deployed and reached bests results in efficiency and operational security in their plants. This article has the goal, from the authors' experiences in development and analysis of lots of management alarms' systems in industry, discuss the reasons of the failure of this systems in reaching better key performing indicators, while operating and managing alarms. It also proposes the adoption of strategies, tools and practices to reach the maximum of operational benefits, when deploying and maintaining these systems.

Keywords: Alarm management; Root cause analysis; Supervisory systems. 


\section{INTRODUÇÃO}

Após publicações tais como a ANSI/ISA 18.2 e EEMUA 191, houve um despertar por parte da indústria sobre a questão da efetividade de seu sistema de alarme em prevenir situações de risco (seja material, ambiental ou humano) e manter suas plantas no melhor estado de eficiência operacional possível. Normalmente, após alguma investigação da real condição dos alarmes de suas plantas, estas empresas identificam que estão bem aquém das recomendações definidas nestes padrões, e partem então para a etapa de implantação de sistemas de gerenciamento de alarmes sem, no entanto, realizar um estudo mais aprofundado dos conceitos defendidos nessas normas. Neste sentido, acabam por adotar soluções mais orientadas a ferramentas genéricas sem se basear em um consistente processo de gerenciamento propriamente dito. O problema dessa abordagem é que, por fazer apenas diagnósticos e não ter um processo que permita agir adequadamente, os resultados esperados na melhoria operacional e de segurança não são atingidos, deixando a planta quase na mesma condição de antes. Além disso, mesmos nas indústrias onde existe a preocupação em aplicar os estágios previstos do gerenciamento de alarmes, verifica-se que bons resultados são atingidos a curto e médio prazo, porém deixam a desejar a longo prazo. Isto se deve a várias falhas na condução do processo de gestão, o que leva o sistema a apresentar condições de ineficiência operacional por não conseguir responder às mudanças que inevitavelmente ocorrem no ambiente industrial.

Diante de tal problema, o presente trabalho visa fazer um rápido apanhado dos principais conceitos e práticas defendidas nas normas e, por meio da análise de vários casos práticos observados pelos autores, identificar os principais problemas na implantação do gerenciamento de alarmes. Pretende-se ainda propor um modelo de gestão que permita a adequada execução dos processos quanto ao uso de ferramentas que suportem eficazmente a monitoração, avaliação e suporte a decisão em tais sistemas.

\subsection{Definição de Alarme}

Segundo ANSI/ISA [1], um alarme é um anúncio iniciado por uma condição de mau funcionamento de equipamento, desvio de processo ou condição anormal que requer uma ação do operador. Apesar deste conceito ser bastante claro e objetivo, verificamos que um dos maiores equívocos encontrados em sistemas de alarmes é quantidade de anúncios que não seguem esta definição. $O$ que frequentemente encontramos nos sistemas é o elevado número de anúncios que não deveriam ter uma ação do operador e, portanto, deveriam ser tratados apenas como um evento do sistema. E se é um evento, este não deveria competir pela atenção do operador como os outros alarmes merecedores de resposta.

Ainda, segundo EEMUA [2], para que os alarmes cumpram seu papel eles devem apresentar as seguintes características:

Relevância - informações irrelevantes ou espúrias devem ser ignoradas;

Singularidade - o mesmo alarme não deve apresentar entradas duplicadas;

Precisão - nenhum alarme deve ser apresentado com muita antecedência a sua resposta ou muito tarde para que a mesma seja executada;

Importância - todo alarme deve possuir uma prioridade no tratamento;

- Clareza - suas informações e ações de resposta devem ser de fácil compreensão; 
Diagnóstico - todo alarme deve identificar o problema ocorrido;

Consultivo - o conjunto de ações a serem tomadas deve estar disponível;

$\square$ Focado - apresentar somente as informações mais importantes para o correto funcionamento da planta naquele estado.

A importância de um sistema de alarme bem concebido é tamanha que, segundo Nimmo [3], podem ajudar o operador a impedir que uma situação anormal se propague ou um distúrbio venha ocorrer. Os benefícios incluem:

Aumento da segurança

Redução de incidentes ambientais

Aumento da produção

Melhoria da qualidade

Redução dos custos

Um sistema de alarmes mal projetado pode, além de levar a prejuízos de ordens econômicas, causar severos acidentes de ameaça à vida e ao ambiente, como o caso da refinaria Milford Haven da Texaco em 1994 [4].

Desta forma, dada a importância do assunto, houve um esforço considerável por parte da indústria em identificar as melhores práticas para concepção de sistemas de alarmes. Dentre esses esforços, estão a EEMUA 191 e ISA 18.2. A primeira é mais focada no fator da usabilidade dos sistemas de alarmes e na definição de métricas de desempenho. A segunda, a ISA 18.2, aborda questões mais relacionadas aos estágios de gestão do sistema de alarme.

\subsection{Gerenciamento de Alarmes}

Uma das recomendações de tais estudos é que os sistemas de alarmes devem ser suportados por processos que garantam o correto funcionamento dos alarmes em todo seu ciclo de vida.

Segundo a norma [5], sistemas de alarmes efetivos são aqueles bem projetados, implementados, operados e mantidos. Um gerenciamento de alarmes é um conjunto de práticas e processos que asseguram um sistema efetivo. Ainda segundo este padrão, o gerenciamento de alarmes pode ser compreendido conforme o seguinte modelo de estágios do ciclo de vida: 


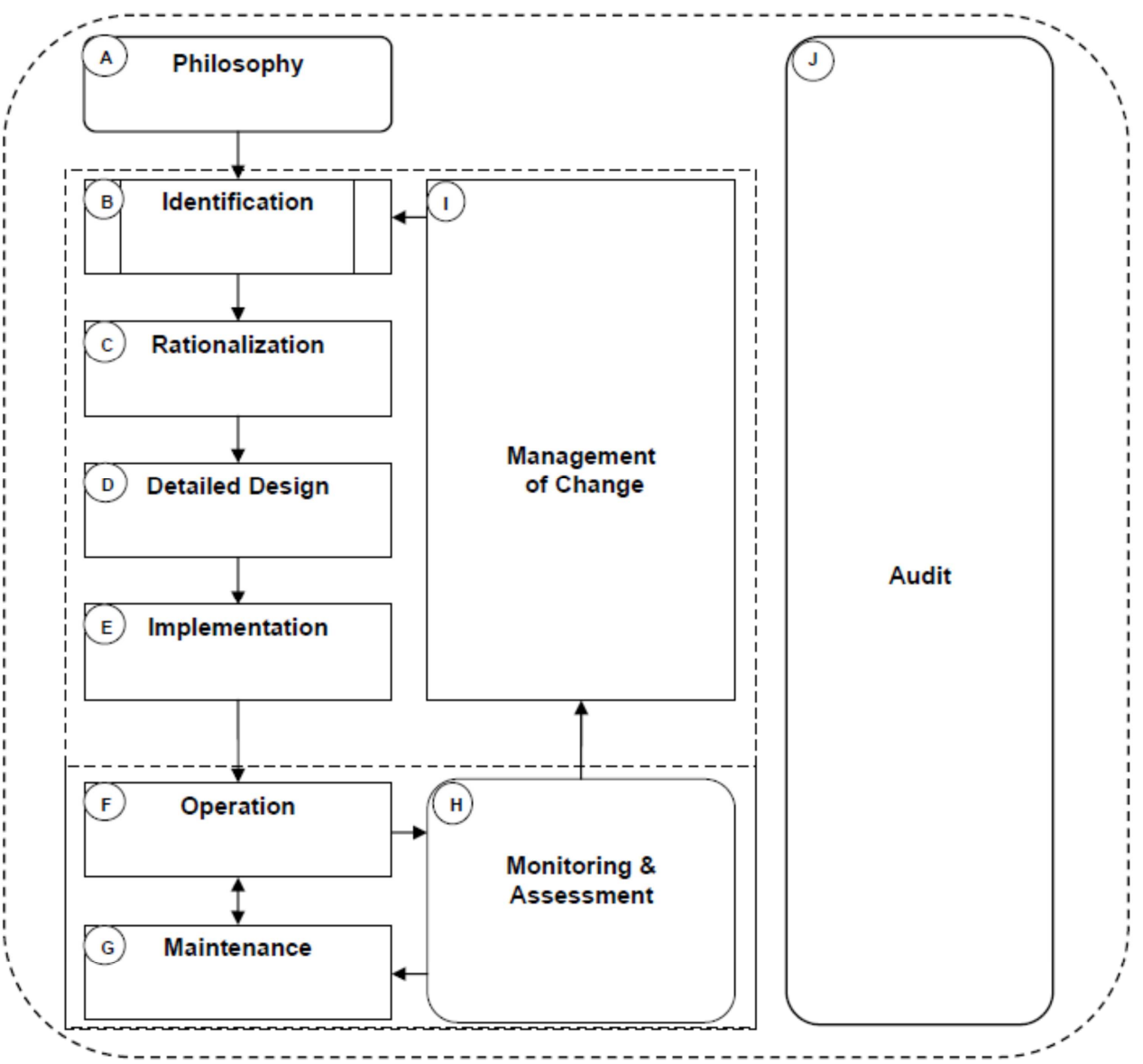

Figura 1. Ciclo de vida do gerenciamento de alarmes

Cada estágio ou processo do ciclo de vida do gerenciamento de alarmes é entendido conforme abaixo:

A. Filosofia de alarmes:

O objetivo deste estágio é elaborar um documento, que defina diretrizes para o gerenciamento de alarmes da organização, por meio de todas as fases do ciclo de vida. Deve conter as regras para a identificação, classificação e priorização de alarmes, utilização de cores para indicar um alarme, e a gestão das alterações nas configurações. Deve também estabelecer parâmetros de desempenho, tais como a carga de alarmes aceitável para o operador. Para as novas instalações, a filosofia de alarmes deve ser totalmente definida e aprovada antes do comissionamento.

B. Identificação e Racionalização:

Neste estágio é feita a identificação e racionalização de um conjunto mínimo de alarmes necessários para manter o processo seguro e sob controle. Racionalização implica uma revisão e justificativa de potenciais alarmes para garantir que eles cumpram os requisitos e a definição de um alarme conforme definido no documento de filosofia de alarmes. Este processo envolve também a documentação da prioridade de cada alarme, classificação, limites, causas, consequências e ações corretivas no banco de dados de alarmes.

C. Projeto detalhado:

Inclui o projeto base de alarme, determinação de parâmetros como banda morta, projetos avançados, utilização de estado do processo ou equipamento para supressão automática de alarme, e projeto de $\mathrm{HMI}$, mostrando o alarme para a operação para que possam efetivamente detectar, diagnosticar e responder a ele. 
D. Implantação, operação e manutenção:

A norma descreve as práticas e procedimentos para a colocar um alarme em operação, utilizá-lo, e retirá-lo de serviço para reparo, substituição ou teste. Exigências e recomendações para treinamento e teste são definidos, bem como as ferramentas que devem estar à disposição do operador para trabalhar com alarmes (tais como supressão de alarme). A norma descreve os procedimentos que devem ser seguidos para retirar um alarme de serviço, incluindo a documentação de justificativa para a remoção, as informações relativas aos alarmes provisórios, procedimentos especiais de manipulação, assim como os testes que são necessários de serem realizados antes de se colocar o alarme novamente em serviço.

E. Acompanhamento e Avaliação:

Um sistema de alarme não monitorado é um sistema danificado. Monitorar o desempenho e compará-lo com as métricas chaves tais como as do padrão é uma atividade fundamental no ciclo de vida.

F. Gerenciamento da Mudança:

Gestão da mudança implica a utilização de procedimentos para garantir que modificações no sistema de alarme passem por revisões e sejam aprovadas antes da implementação.

G. Auditoria:

Fase de auditoria do ciclo de vida de alarme é focada principalmente na revisão periódica dos processos de trabalho e desempenho dos sistemas de alarme. $O$ objetivo é manter sua integridade em todo o seu ciclo de vida para identificar áreas de melhoria.

\title{
1.3 Situação do Gerenciamento de Alarmes na Indústria
}

Apesar dos padrões e recomendações de boas práticas serem bastante completas, o gerenciamento de alarmes é um processo complexo e para atingir resultados significativos é necessário ter disciplina e comprometimento das organizações que 0 adotam.

A maioria dos projetos de gerenciamento de alarmes, segundo Nimmo [3], apresenta certo grau de sucesso devido à natureza do problema, mas poucos alcançam a meta inicial de melhorar significativamente os sistemas de alarmes. O motivo é normalmente causado pela estimativa ruim do tempo necessário para solucionar este tipo de problema e a complexidade da relação em cadeia da gestão de alarmes, assim como as implicações em outros sistemas, tais como treinamento, interface homem máquina, etc. Os motivos de falha em projetos de gerenciamento de alarmes incluem:

\author{
Objetivos modestos \\ Responsabilidades não claras \\ Plano de trabalho inexistente ou pobre \\ Vários objetivos \\ Decisões de processo confusas \\ Baixa expectativa \\ Desconhecimento do processo \\ Falta de padronização \\ Objetivos não claros ou não mensuráveis \\ Programas de revisão inexistente ou ineficiente \\ Adoção de rotinas ultrapassadas
}


Falta de comprometimento dos envolvidos

Segundo O'Brien et al. [6], a situação do gerenciamento de alarmes presente nas indústrias fica evidenciada da seguinte maneira:

"Gerenciamento de alarmes é um dos aspectos mais subestimados e subutilizados do processo de automação, onde muitas plantas ainda utilizam a filosofia de gerenciamento de alarmes desenvolvida no início de sua construção. Uma vez que os sistemas de alarme se tornam menos efetivos, eles reduzem a efetividade de toda automação."

\section{MATERIAIS E MÉTODOS}

A metodologia empregada neste trabalho consistiu em fazer a comparação da classificação de desempenho do sistema de alarme de uma planta industrial antes e depois da implantação do gerenciamento de alarmes. A planta industrial mencionada é de uma unidade de britagem de uma mineradora do estado de Minas Gerais.

Ambas situações foram comparadas aos indicadores de desempenho recomendadas pelas normas. $O$ objetivo de tal abordagem foi verificar o real impacto na implantação do gerenciamento de alarmes no desempenho da operação.

Para os casos nas quais as medições foram bem abaixo do esperado após implantação do gerenciamento de alarmes, foi feita uma entrevista com os envolvidos na manutenção e gestão do sistema e uma conduzida uma investigação baseada na técnica de causa raiz.

\subsection{Classificações de Desempenho}

A classificação de desempenho foi baseada na análise de dois índices de desempenho a saber:

1. Número máximo de alarmes por hora

2. Número médio de alarmes por hora

Esses dois índices foram gerados usando uma ferramenta de análise de sistemas de alarmes.

A partir da combinação destes dois índices em um único gráfico, conforme proposto por EEMUA [2], podemos classificar o sistema conforme figura abaixo:

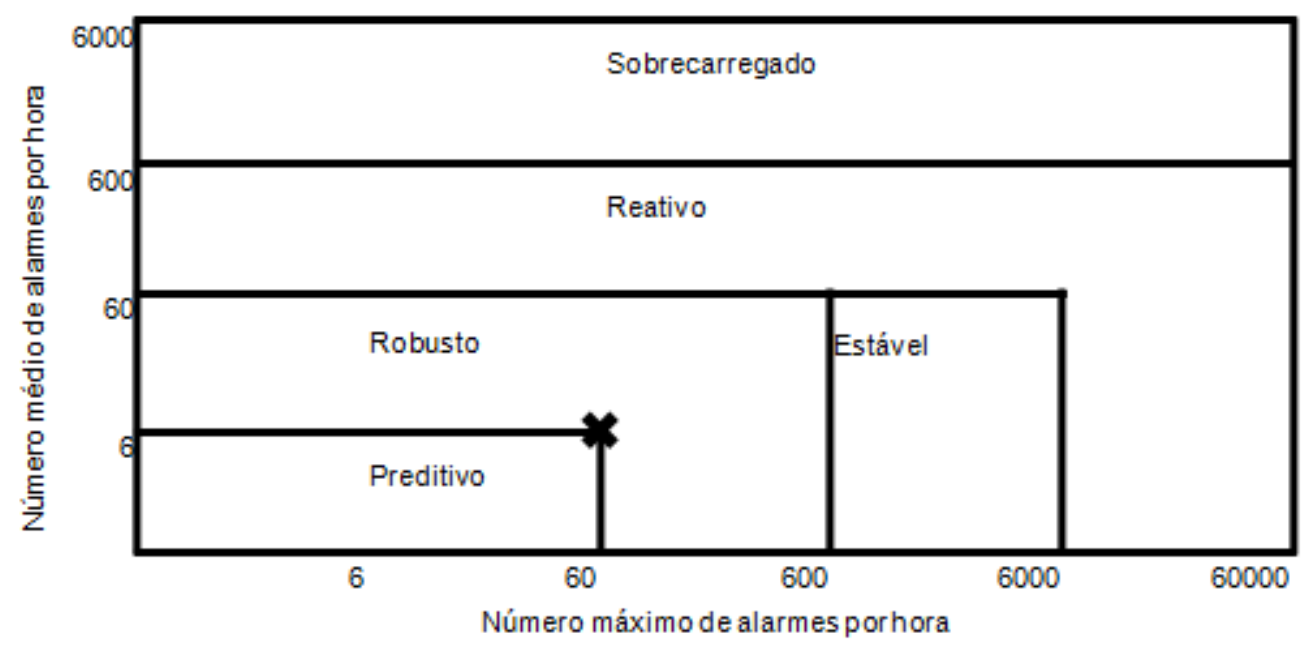

Figura 2. Classificação ideal de um sistema de alarme, segundo EEMUA [2] 
Os níveis possíveis de classificação são os seguintes:

a) Sobrecarregado: Alta taxa contínua de alarmes. Condição altamente insegura para a produção e segurança do processo. Operação não consegue distinguir alarmes críticos e de alarmes baixa relevância.

b) Reativo: Nível inicial da maioria das plantas. Operação ainda não plenamente consegue tratar alarmes em condições normais da planta.

c) Estável: Operação já consegue responder bem em condições normais, mas ainda é insuficiente em condições de emergências ou distúrbios gerados de paradas/partidas da planta.

d) Robusto: Operação consegue tratar alarmes em tempo ideal em todos as situações de operação da planta, incluindo emergências e distúrbio de paradas/partidas.

e) Preditivo: Operação tem um sistema de alarme eficiente o suficiente para tratar preditivamente a maior parte das situações operacionais. Deve ser encarado como classificação alvo de todo gerenciamento de alarmes.

\subsection{Método Causa Raiz}

O método de causa raiz, desenvolvida por Taiichi Ohno, pai do Sistema de Produção Toyota, consiste em formular a pergunta "Por quê" cinco vezes para compreender o que aconteceu (a causa-raiz). Nada impede, porém, que mais (ou menos) do que cinco perguntas sejam feitas. O número cinco vem da observação de Ono de que essa quantidade costuma ser suficiente para se chegar a causa raiz.

Este método engloba um conjunto específico de etapas, com instrumentos associados, para encontrar a causa primária do problema, de modo que você pode:

1. Determinar o que aconteceu.

2. Determinar por que isso aconteceu.

3. Descobrir o que fazer para reduzir a probabilidade de que isso vai acontecer novamente.

\section{RESULTADOS E DISCUSSÃO}

\subsection{Resultados dos Indicadores de Desempenho}

Tabela 1. Comparativo de desempenho (situação antes e depois do gerenciamento de alarme)

\begin{tabular}{|l|c|c|}
\hline Índice de desempenho & $\begin{array}{l}\text { Antes do gerenciamento } \\
\text { de alarmes }\end{array}$ & $\begin{array}{l}\text { Depois do gerenciamento } \\
\text { de alarmes }\end{array}$ \\
\hline $\begin{array}{l}\text { Número máximo de } \\
\text { alarmes por hora }\end{array}$ & 47.460 & 4.039 \\
\hline $\begin{array}{l}\text { Número médio de } \\
\text { alarmes por hora }\end{array}$ & 580 & 48 \\
\hline
\end{tabular}

Com base nos nestes índices, podemos traçar a classificação antes e depois da adoção do gerenciamento de alarmes: 


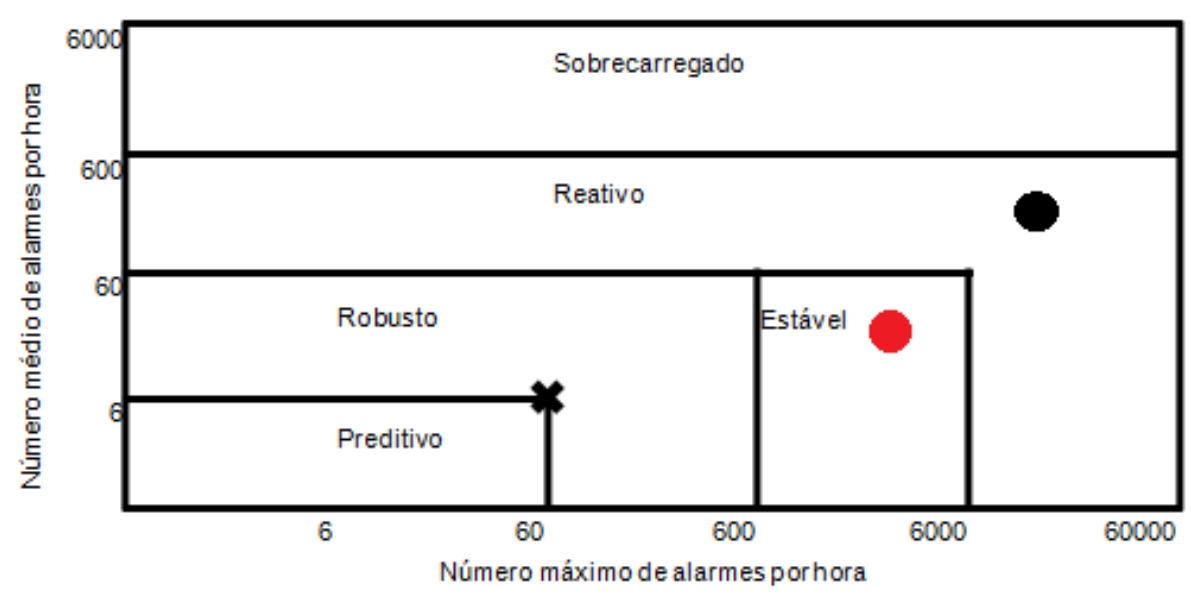

Figura 3. Comparativo de classificação do sistema de alarme (situação antes e depois)

Sendo que:

- Símbolo para representar a situação após implantação do gerenciamento de alarmes.

- Símbolo para representar a situação antes da implantação do gerenciamento de alarmes.

\subsection{Análises de Causa Raiz}

Após análises de causa raiz, foram identificadas várias dificuldades e más práticas comuns principalmente na forma de conduzir o processo de gestão de alarmes. Agrupamos os principais erros associados a cada processo conforme a seguir:

1. Definição de Filosofia de alarmes

- Documento de filosofia incompleto.

- Falta de definição de papel e responsabilidades

- Ausência de um processo que consiga manter o documento de filosofia atualizado conforme as necessidades do processo.

2. Identificação de alarmes/Racionalização de alarmes

- Técnicas não adequadas de identificação

- Critérios de priorização subjetivos

- Muitos níveis de priorização

- Falta de envolvimento de representantes de todos os segmentos de usuários e partes interessadas no sistema de alarmes

- Não considerar os cenários de supressão de alarmes

- Situação de partidas/paradas de plantas não devidamente identificados

3. Detalhamento de projeto/Implementação

- Ausência de recursos chave para a adequada operação do sistema

4. Operação/Manutenção

- Resistência cultural da operação

- Manutenção sem controle e registros de mudanças

5. Monitoramento e avaliação

- Falta de um processo de análise de relatórios das ferramentas

6. Gestão de mudanças

- Falta de responsável para tratar devidamente as mudanças

- Falha ou analisar todo o processo $\rightarrow$ revisão do documento de filosofia de alarmes 
7. Auditoria

- Ausência de processo que analise se a gestão de alarmes está gerando valor para organização.

\section{CONCLUSÃO}

O gerenciamento de alarmes pode melhorar consideravelmente a qualidade da resposta da operação às situações anormais de processo, trazendo assim melhorias para o processo e segurança da planta. No entanto, como pudemos verificar pelos índices de desempenho, mesmo após implantação do gerenciamento de alarmes, o sistema atingiu uma classificação mediana, ainda aquém da sugerida pelos padrões das normas. A análise de causa raiz demonstra que boa parte dos problemas que impedem que o sistema alcance níveis melhores de classificação são relacionados à condução e monitoramento dos processos definidos para o gerenciamento de alarmes. Desta forma, estratégias que implementam controles mais sofisticados sobre os processos podem gerar classificações próximas do ponto ideal definido pelas normas e, consequentemente, melhorando o resultado operacional das plantas industriais.

\section{REFERÊNCIAS}

1. ANSI/ISA. Management of Alarm Systems for the Process Industries. SP 18.2. 2009.

2. Engineering Equipment Materials Users' Association. Alarm Systems A Guide to Design, Management and Procurement. EEMUA Publication 191, second edition, London, 2007.

3. Nimmo, I. The Importance of Alarm Management Improvement Project. INTERKAMA. 1999.

4. Bransby, M., Jenkinson, J.. The management of alarm systems. HSE Contract Research Report 166. Suffolk, UK: HSE Books, 1998

5. O'Brien, L., O.,Woll, D. Alarm Management Strategies. ARC Strategies. USA. November 2004. 